\title{
Checklist dos Plecoptera (Insecta) do Estado de São Paulo, Brasil
}

\section{Claudio Gilberto Froehlich ${ }^{1,2}$}

${ }^{1}$ Departamento de Biologia, Faculdade de Filosofia, Ciências e Letras, Universidade de São Paulo - USP Av. Bandeirantes, $n$. 3900, CEP 14040-901, Ribeirão Preto, SP, Brasil

${ }^{2}$ Autor para correspondência: Claudio Gilberto Froehlich,e-mail: cgfroeh@usp.br

FROEHLICH, C.G. Checklist of Plecoptera from São Paulo State, Brazil. Biota Neotrop. 11(1a): http://www. biotaneotropica.org.br/v11n1a/en/abstract?inventory+bn0291101a2011.

Abstract: In 1999 the number of species of stoneflies known from the State of São Paulo was 40. In 2009, during the first ten years of the BIOTA/FAPESP program 16 new species were added, bringing the total to 56 species. Keywords: Plecoptera, biodiversity of the State of São Paulo, BIOTA/FAPESP Program.

Number of species: in the world: 3,500 (Fochetti \& Tierno de Figueroa 2008), in Brazil: 140, estimated in São Paulo State: 80.

FROEHLICH, C.G. Checklist dos Plecoptera (Insecta) do Estado de São Paulo, Brasil. Biota Neotrop. 11(1a): http://www.biotaneotropica.org.br/v11n1a/pt/abstract?inventory+bn0291101a2011.

Resumo: Em 1999, conheciam-se 40 espécies de plecópteros do Estado de São Paulo. Em 2009, após os 10 primeiros anos do Programa BIOTA/FAPESP, 16 espécies foram descritas, elevando o total para 56 espécies. Palavras-chave: Plecoptera, biota paulista, Programa BIOTA/FAPESP.

Número de espécies: no mundo: 3.500 (Fochetti \& Tierno de Figueroa 2008), no Brasil: 140, estimadas no Estado de São Paulo: 80. 


\section{Introdução}

Os Plecoptera constituem uma ordem relativamente pequena de insetos caracterizados por terem ninfas aquáticas e adultos do ambiente aéreo. Sua posição filogenética é discutida, podendo ser o grupo-irmão dos Neoptera restantes ou o grupo-irmão dos Polyneoptera restantes (Zwick 2009). São cosmopolitas, faltando em muitas ilhas oceânicas e na Antártica. São divididos em 16 famílias, das quais 6 ocorrem na Região Neotropical e apenas duas (Gripopterygidae e Perlidae) no Brasil. Ambas ocorrem no Estado de São Paulo.

Adultos de Plecoptera são encontrados próximos dos rios onde vivem as ninfas. Estas preferem águas limpas e, geralmente, de temperaturas mais baixas, razão porque são mais frequentes em áreas montanhosas.

Foi publicado recentemente um livro sobre a ordem Plecoptera na América Latina (Stark et al. 2009), onde se encontram chaves para famílias e gêneros e informações gerais sobre os táxons.

\section{Metodologia}

Para elaboração da lista das espécies do Estado de São Paulo (Tabela 1) foram consultados:

Froehlich (2010): Catalogue of Neotropical Plecoptera. Illiesia, 6 (12): 118-205.

Lecci e Froehlich (2006): Plecoptera. http://sites.ffclrp.usp.br/ aguadoce/plecoptera/plecindex.htm. In: Levantamento e biologia de Insecta e Oligochaeta aquáticos de sistemas lóticos do Estado de São Paulo. http://sites.ffclrp.usp.br/aguadoce (última atualização: junho 2008).

\section{Resultados e Discussão}

Froehlich (1999), na obra sobre a Biodiversidade do Estado de São Paulo, listou 40 espécies conhecidas do estado. Este número subiu para 56 no presente artigo. $\mathrm{O}$ aumento relativamente pequeno de 16 espécies deve-se, por um lado, ao fato de os Plecoptera já serem melhor conhecidos que outras ordens de insetos aquáticos e, por outro lado, à importância dada à ecologia durante o desenvolvimento dos projetos incluídos no Programa BIOTA/FAPESP (Processos 1998/05073-4 e 2003/10517-9), como pode ser visto na lista de artigos publicados e de dissertações ou teses defendidas. Essa ênfase decorreu da percepção de que trabalhos ecológicos sobre a fauna de rios do Estado de São Paulo, em particular os referentes a insetos, eram escassos. Com o crescente uso da biota fluvial, em especial os insetos, em programas de biomonitoramento da qualidade da água e na elaboração de estudos ou relatórios de impactos ambientais, os participantes dos dois projetos de levantamento e biologia de insetos e outros invertebrados junto ao Programa BIOTA/FAPESP sentiram a necessidade de, além dos levantamentos faunísticos, iniciarem um programa de estudos ecológicos.

Os dois projetos contribuíram, sem dúvida, para um conhecimento muito maior da fauna dos macroinvertebrados de águas correntes no Estado, em termos de composição faunística, distribuição geográfica e ecologia. Em ecologia foram abordadas pesquisas de estrutura de comunidades, distribuição espacial em diferentes escalas, distribuição temporal, e influência de fatores ambientais, inclusive daqueles causados pelos impactos antropogênicos. No primeiro projeto foram estudados os Crustacea, Mollusca, Insecta e Oligochaeta e, no segundo, Oligochaeta e Insecta. As ordens de insetos consideradas foram os Ephemeroptera, Odonata, Plecoptera, Coleoptera, Trichoptera e Diptera (famílias Chironomidae e Simuliidae).

Tabela 1. Lista das espécies de Plecoptera do Estado de São Paulo.

Table 1. List of species of stoneflies from the State of São Paulo.

\begin{tabular}{|c|c|c|c|}
\hline $\mathbf{N}^{\circ}$ & Espécie & Descritor & Espécimes em Coleção Científica \\
\hline \multicolumn{4}{|c|}{ Família Gripopterygidae } \\
\hline 1 & Gripopteryx cancellata & (Pictet 1841) & 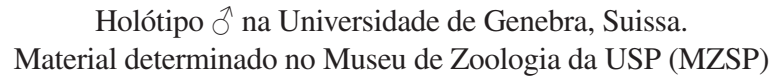 \\
\hline 2 & Gripopteryx coruja & Froehlich 1993 & Holótipo $\widehat{\jmath}$ e parátipos no MZSP \\
\hline 3 & Gripopteryx flinti & Froehlich 1993 & Holótipo $\widehat{\jmath}$ e parátipos no MZSP \\
\hline 4 & Gripopteryx garbei & Navás 1936 & Holótipo + e material determinado no MZSP \\
\hline 5 & Gripopteryx juetah & Froehlich 1990 & Holótipo $\widehat{o}$ e parátipos no MZSP \\
\hline 6 & Gripopteryx pilosa & Froehlich 1990 & Holótipo $\widehat{\jmath}$ e parátipos no MZSP \\
\hline 7 & Gripopteryx pinima & Froehlich 1993 & Holótipo $\widehat{O}$ e parátipos no MZSP \\
\hline 8 & Gripopteryx reticulata & Brauer 1866 & $\begin{array}{c}\text { Holótipo ổ no Museu de História Natural de Viena, Áustria. } \\
\text { Material determinado no MZSP }\end{array}$ \\
\hline 9 & Guaranyperla guapiara & Froehlich 2001 & Holótipo $q$ e parátipos no MZSP \\
\hline 10 & Guaranyperla nitens & Froehlich 2001 & Holótipo ? no MZSP \\
\hline 11 & Paragripopteryx anga & Froehlich 1969 & Holótipo $\widehat{\jmath}$ e parátipos no MZSP \\
\hline 12 & Paragripopteryx blanda & Froehlich 1969 & Holótipo $\widehat{\jmath}$ e parátipos no MZSP \\
\hline 13 & Paragripopteryx delicata & Froehlich 1994 & Holótipo $\widehat{\jmath}$ e parátipos no MZSP \\
\hline 14 & Paragripopteryx egena & Froehlich 1994 & Holótipo $\widehat{\jmath}$ e parátipos no MZSP \\
\hline 15 & Paragripopteryx guardae & Froehlich 1994 & Holótipo $\widehat{\jmath}$ e parátipos no MZSP \\
\hline 16 & Paragripopteryx hamata & Froehlich 1994 & Holótipo $\widehat{~}$ e parátipos no MZSP \\
\hline 17 & Paragripopteryx klapaleki & Enderlein 1909a & Holótipo perdido. Material determinado no MZSP \\
\hline 18 & Paragripopteryx merui & Froehlich 1994 & Holótipo $\widehat{\jmath}$ e parátipos no MZSP \\
\hline 19 & Tupiperla eleonorae & (Froehlich 1994) & Holótipo đ̊ e parátipos no MZSP \\
\hline 20 & Tupiperla gracilis & (Burmeister 1839) & $\begin{array}{c}\text { Holótipo } \widehat{\jmath} \text { na Universidade de Halle, Alemanha. } \\
\text { Material determinado no MZSP }\end{array}$ \\
\hline 21 & Tupiperla illiesi & Froehlich 1998 & Holótipo $\widehat{\jmath}$ e parátipos no MZSP \\
\hline
\end{tabular}


Tabela 1. Continuação...

\begin{tabular}{|c|c|c|c|}
\hline $\mathbf{N}^{\circ}$ & Espécie & Descritor & Espécimes em Coleção Científica \\
\hline 22 & Tupiperla modesta & Froehlich 1998 & Holótipo đ̃ e parátipos no MZSP \\
\hline 23 & Tupiperla reichardti & Froehlich 1998 & Holótipo đo e parátipos no MZSP \\
\hline 24 & Tupiperla robusta & Froehlich 1998 & Holótipo đ e parátipos no MZSP \\
\hline 25 & Tupiperla tessellata & (Brauer 1866) & $\begin{array}{c}\text { Holótipo } 9 \text { no Museu de História Natural de Viena, Áustria. } \\
\text { Material determinado no MZSP }\end{array}$ \\
\hline 26 & Tupiperla umbya & Froehlich 1998 & Holótipo đ e parátipos no MZSP \\
\hline \multicolumn{4}{|r|}{ Família Perlidae } \\
\hline 1 & Anacroneuria boraceiensis & Froehlich 2004 & Holótipo đ̊ e parátipos no MZSP \\
\hline 2 & Anacroneuria debilis & (Pictet 1841) & $\begin{array}{c}\text { Holótipo } ð \text { no Museu da Universidade Humboldt em Berlim, Alemanha. } \\
\text { Material determinado no MZSP }\end{array}$ \\
\hline 3 & Anacroneuria flintorum & Froehlich 2002 & Holótipo $\precsim$ e material determinado no MZSP \\
\hline 4 & Anacroneuria iporanga & Bispo \& Froehlich 2004 & Holótipo $\precsim$ e parátipo $\precsim$ no MZSP \\
\hline 5 & Anacroneuria itajaimirim & Bispo \& Froehlich 2004 & Holótipo đ̊ e parátipos no MZSP \\
\hline 6 & Anacroneuria paulina & (Navás 1936) & Holótipo $\bigcirc$ e material determinado no MZSP \\
\hline 7 & Anacroneuria petersi & Froehlich 2002 & Holótipo đe parátipo đ̊ no MZSP \\
\hline 8 & Anacroneuria polita & (Burmeister 1839) & $\begin{array}{l}\text { Holótipo đ̂ na Universidade de Halle, Alemanha. } \\
\text { Material determinado no MZSP }\end{array}$ \\
\hline 9 & Anacroneuria stanjewetti & Froehlich 2002 & $\begin{array}{l}\text { Holótipo ô e parátipos na California Academy of Sciences, São Francisco, } \\
\text { California, Estados Unidos }\end{array}$ \\
\hline 10 & Anacroneuria subcostalis & Klapálek 1921 & Dois síntipos em Viena, Austria. Material determinado no MZSP \\
\hline 11 & Anacroneuria toriba & Froehlich 2002 & $\begin{array}{c}\text { Holótipo }{ }^{\lambda} \text { no National Museum of Natural History, Washington, DC, Esta- } \\
\text { dos Unidos. Material determinado no MZSP }\end{array}$ \\
\hline 12 & Anacroneuria tupi & Bispo \& Froehlich 2004 & Holótipo $\precsim$ e parátipos no MZSP \\
\hline 13 & Anacroneuria uyara & Froehlich 2002 & 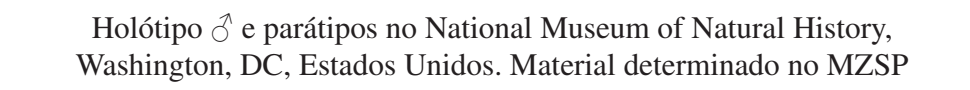 \\
\hline 14 & Anacroneuria vanini & Froehlich 2004 & Holótipo đ e parátipos no MZSP \\
\hline 15 & Kempnyia auberti & Froehlich 1996 & Holótipo $\widehat{\delta}$ e parátipo $\widehat{\partial}$ no MZSP \\
\hline 16 & Kempnyia barbiellinii & (Navás 1925) & Holótipo perdido \\
\hline 17 & Kempnyia colossica & (Navás 1934) & $\begin{array}{c}\text { Holótipo }+ \text { em Müncheberg (Deutsches Entomologisches Institut), } \\
\text { Alemanha. Material determinado no MZSP }\end{array}$ \\
\hline 18 & Kempnyia flava & Klapálek 1916 & $\begin{array}{c}\text { Lectótipo } q \text { no Museu de História Natural de Vienna, Áustria. } \\
\text { Material determinado no MZSP }\end{array}$ \\
\hline 19 & Kempnyia gracilenta & Enderlein 1909 & 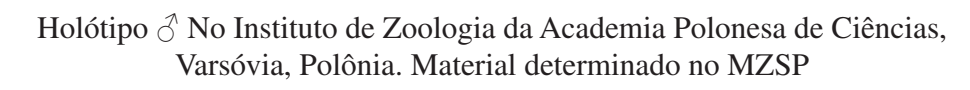 \\
\hline 20 & Kempnyia jatim & Froehlich 1988 & Holótipo đ e parátipos no MZSP \\
\hline 21 & Kempnyia mirim & Froehlich 1984 & Holótipo đ e parátipos no MZSP \\
\hline 22 & Kempnyia neotropica & $\begin{array}{l}\text { (Jacobson \& Bianchi } \\
\text { 1905) }\end{array}$ & $\begin{array}{c}\text { Holótipo đ̊ no Museu da Universidade Humboldt em Berlim, Alemanha. } \\
\text { Material determinado no MZSP }\end{array}$ \\
\hline 23 & Kempnyia obtusa & Klapálek 1916 & $\begin{array}{c}\text { Holótipo } \widehat{C} \text { no Museu de História Natural de Viena, Áustria. } \\
\text { Material determinado no MZSP }\end{array}$ \\
\hline 24 & Kempnyia petersorum & Froehlich 1996 & Holótipo đ e parátipos no MZSP \\
\hline 25 & Kempnyia reichardti & Froehlich 1984 & Holótipo $\widehat{o}$ e parátipos no MZSP \\
\hline 26 & Kempnyia serrana & (Navás 1936) & Holótipo $\widehat{o}$ e material determinado no MZSP \\
\hline 27 & Kempnyia tamoya & Froehlich 1984 & Holótipo $\precsim$ no MZSP \\
\hline 28 & Kempnyia umbrina & Froehlich 1988 & Holótipo đ̊ e parátipos no MZSP \\
\hline 29 & Kempnyia vanini & Froehlich 1988 & Holótipo $\widehat{o}$ e parátipos no MZSP \\
\hline 30 & Macrogynoplax veneranda & Froehlich 1984 & Holótipo $\widehat{o}$ e material determinado no MZSP \\
\hline
\end{tabular}




\section{Comentários Sobre a Lista, Riqueza do Estado Comparada com Outras Regiões}

O conhecimento taxonômico dos insetos aquáticos do Brasil, exceto os de interesse médico, é muito incipiente. A situação é melhor nos Estados de São Paulo, em grande parte devida ao Programa BIOTA/FAPESP, do Rio de Janeiro, pelos trabalhos desenvolvidos por pesquisadores da Universidade Federal do Rio de Janeiro e do Instituto Oswaldo Cruz e do Amazonas, por pesquisadores do INPA. O Estado de São Paulo é o que tem o maior número de espécies conhecidas de Plecoptera, 56 até 2009. O segundo é o Estado do Rio de Janeiro, com 32 e o terceiro é Santa Catarina, com 23 espécies. Dez estados brasileiros, principalmente do Nordeste, e o Distrito Federal não têm registros de Plecoptera.

\section{Principais Avanços Relacionados ao Programa BIOTA/FAPESP}

Como citado acima, foram adicionadas, até 2009, 16 espécies de Plecoptera à fauna do Estado de São Paulo. Os estudos ecológicos realizados pela equipe dos projetos ligados ao Programa BIOTA/ FAPESP, incluindo os Plecoptera, representaram praticamente o início do desenvolvimento dessa área de pesquisa no Estado.

\section{Principais Grupos de Pesquisa}

1)Laboratório de Entomologia Aquática, do Departamento de Biologia da FFCL de Ribeirão, Preto, USP, liderado por Claudio Gilberto Froehlich.

2)Laboratório de Biologia Aquática, UNESP, campus de Assis, liderado por Pitágoras da Conceição Bispo.

\section{Principais Acervos}

O principal acervo de Plecoptera encontra-se no Museu de Zoologia da USP, onde se encontra depositado o material já estudado dos projetos BIOTA e de projetos anteriores. Material em estudo encontra-se no Laboratório de Entomologia Aquática, Departamento de Biologia, FFCLRP, USP de Ribeirão Preto e no Laboratório de
Biologia Aquática, UNESP, campus de Assis, onde é também mantida uma coleção de referência.

\section{Principais Lacunas do Conhecimento}

Grandes áreas do Estado de São Paulo permanecem inexploradas quanto aos insetos aquáticos. Com o tempo, essas áreas deverão ser amostradas.

\section{Perspectivas de Pesquisa para os Próximos 10 Anos}

Os estudos ecológicos sobre os insetos aquáticos devem ser incrementados pela importância que têm adquirido com a crescente preocupação pela qualidade ambiental e necessidade de biomonitoramento. Ao mesmo tempo, as pesquisas taxonômicas são fundamentalmente necessárias para as pesquisas ecológicas, além do interesse acadêmico. No caso dos Plecoptera, além de ampliar o conhecimento da ordem, estudos filogenéticos, já iniciados, devem ter prosseguimento.

\section{Referencias Bibliográficas}

FOCHETTI, R. \& TIERNO DE FIGUEROA, J.M. 2008. Global diversity of stoneflies (Plecoptera; Insecta) in freshwater. Hydrobiologia 595:365-377. http://dx.doi.org/10.1007/s10750-007-9031-3

FROEHLICH, C.G. 1999. Insetos Plecópteros. In: Biodiversidade do Estado de São Paulo. Invertebrados de água doce. (D. Ismael, C. Valenti, T. MatsumuraTundisi \& O. Rocha, eds.). FAPESP, São Paulo, v.4, cap.23, p.159-160.

FROEHLICH, C.G. 2010. Catalogue of Neotropical Plecoptera. Illiesia 6(12):118-205.

LECCI, L.S. \& FROEHLICH, C.G. 2006. Plecoptera. In Levantamento e biologia de Insecta e Oligochaeta aquáticos de sistemas lóticos do Estado de São Paulo. http://sites.ffclrp.usp.br/aguadoce/plecoptera/plecindex. htm: (último acesso em 17/02/2011).

STARK, B.P., FROEHLICH, C. \& ZÚÑIGA, M.C. 2009. South American Stoneflies (Plecoptera). Aquatic Biodiversity in Latin America. Pensoft, Sofia \& Moscow, v.5., 154p.

ZWICK, P. 2009. The Plecoptera - who are they? The problematic placement of stoneflies in the phylogenetic system of insects. Aquat. Ins. 31(Suppl. 1):181-194. http://dx.doi.org/10.1080/01650420802666827

Recebido em 10/05/2010 Versão reformulada recebida em 05/10/2010 Publicado em 15/12/2010 


\section{Apêndices}

Apêndice 1. Lista de teses e dissertações que incluíram a ordem Plecoptera.

Appendix 1. List of PhD theses and MSc dissertations that included the Order Plecoptera.

\section{Teses}

2002 Adriano Sanches Melo: "Estudos sobre estimadores de riqueza de espécies, perturbações experimentais e persistência ao longo de cinco anos em comunidades de macroinvertebrados em riachos.” Orientador: Prof. Dr. Claudio G. Froehlich. Área de Ecologia, UNICAMP. Bolsista FAPESP.

2002 Pitágoras da Conceição Bispo: "Estudo de comunidades de Ephemeroptera, Plecoptera e Trichoptera (EPT) em riachos do Parque Estadual Intervales, Serra de Paranapiacaba, Sul do Estado de São Paulo.” Orientador: Prof. Dr. Claudio G. Froehlich. Área de Zoologia, IBUSP. Bolsista FAPESP.

2003 Vera Lúcia Crisci-Bispo: "Ecologia de imaturos de Ephemeroptera, Plecoptera e Trichoptera (EPT) associados ao substrato rochoso e ao folhiço, e dinâmica de colonização de macroinvertebrados aquáticos em riachos do Parque Estadual Intervales." Orientador: Prof. Dr. Claudio G. Froehlich. Área de Biologia Comparada, FFCLRP-USP. Bolsista CAPES.

2006 Juliano José Corbi: "Influência de diferentes usos da terra sobre os macroinvertebrados aquáticos de córregos: ênfase para o cultivo de cana-de-açúcar em áreas adjacentes." PPG-ERN, UFSCar. Orientadora: Profa. Dra. Susana Trivinho-Strixino.

2008 Marcia Thais Suriano. "Macroinvertebrados bentônicos em córregos de baixa ordem sob diferentes usos do solo no Estado de São Paulo: subsídios para biomonitoramento.” PPG-ERN-UFSCar; Orientadora: Profa. Dra. Alaíde Aparecida Fonseca Gessner.

\section{Dissertações}

2001 Valdelânia Ribeiro de Ribeiro: "Estudo faunístico sobre os Plecoptera em três áreas de preservação ambiental do Estado de São Paulo." Orientador: Prof. Dr. Claudio G. Froehlich. Área de Biologia Comparada, FFCLRP-USP. Bolsista FAPESP. Dissertação defendida em $16 / 10 / 2001$.

2007 Priscilla Kleine. "Macroinvertebrados em córregos da região da Mata Atlântica (Sudeste do Brasil): Influência do cultivo de banana." PPG-ERN, UFSCar, São Carlos, SP, 31/08/2007. Orientadora: Profa. Dra. Susana Trivinho-Strixino.

2008 Márcia Cristina de Paula "Macroinvertebrados em córregos em fragmentos de mata com diferentes estados de conservação no interior do Estado de São Paulo.” PPG-ERN, UFSCar; 06/06/2008. Orientadora: Profa. Dra. Alaíde Aparecida Fonseca Gessner.

2009 Lucas Silveira Lecci. “Sistemática de Gripopteryx (Pictet 1841) (Plecoptera: Gripopterygidae).” PPG-Entomologia, FFCLRP/USP, Ribeirão Preto, SP, 20/03/2009. Orientador: Claudio Gilberto Froehlich.

Apêndice 2. Lista de trabalhos que incluem Plecoptera do Estado de São Paulo (1999-2009).

Appendix 2. List of papers including Plecoptera of São Paulo State (1999-2009).

AVELINO-CAPISTRANO, F.S. \& COSTA, J.M. 2009. Coleção de tipos de Plecoptera (Insecta) do Museu Nacional (UFRJ), Rio de Janeiro, Brasil. Biota Neotrop. 9(4):67-69. http://dx.doi.org/10.1590/S1676-06032009000400007

BISPO, P.C. \& FROEHLICH, C.G. 2004. Perlidae (Plecoptera) from Intervales State Park, São Paulo State, southeastern Brazil. Aquat. Ins. 26(2):97-114. http://dx.doi.org/10.1080/01650420412331325837

BISPO, P.C. \& FROEHLICH, C.G. 2008. Description of the larva and redescription of the adult of Kempnyia neotropica Jacobsen and Bianchi (Plecoptera: Perlidae) with biological notes. Aquat. Ins. 30(1):61-67. http://dx.doi.org/10.1080/01650420701829641

CRISCI-BISPO, V.L., BISPO, P.C. \& FROEHLICH, C.G. 2007a. Ephemeroptera, Plecoptera and Trichoptera assemblages in two Atlantic Rainforest streams, Southeastern Brazil. Rev.Bras. Zool. 24(2):312-318. http://dx.doi.org/10.1590/S0101-81752007000200007

CRISCI-BISPO, V.L., BISPO, P.C. \& FROEHLICH, C.G. 2007b. Ephemeroptera, Plecoptera and Trichoptera assemblages in litter in a mountain stream of the Atlantic Rainforest from Southeastern Brazil. Rev. Brasil. Zool. 24(3):545-551. http://dx.doi.org/10.1590/S0101-81752007000300004

FROEHLICH, C.G. 1999. Insetos Plecópteros. In Biodiversidade do Estado de São Paulo: síntese do conhecimento ao final do século XX. Invertebrados de água doce (D. Ismael, C. Valenti, T. Matsumura-Tundisi, O. Rocha, coords.). FAPESP, São Paulo, v.4, cap.23, p.159-160.

FROEHLICH, C.G. 2001a. Insetos Aquáticos. Em: Intervales. Fundação para a Conservação e a Produção Florestal do Estado de São Paulo. Governo do Estado de São Paulo; Secretaria de Estado do Meio Ambiente, São Paulo, p.181-188.

FROEHLICH, C.G. 2001b. Guaranyperla, a new genus in the Gripopterygidae (Plecoptera). In Trends in Research in Ephemeroptera and Plecoptera (E. Dominguez, ed.). Kluwer Academic; Plenum Publishers, New York, p.379-383.

FROEHLICH, C.G. 2004. Anacroneuria (Plecoptera: Perlidae) from the Boracéia Biological Station, São Paulo State, Brazil. Aquat. Ins. 26(1):53-63. http:// dx.doi.org/10.1076/aqin.26.1.53.35377

FROEHLICH, C.G. 2008. Old species of Neotropical Plecoptera. In International Advances in the Ecology, Zoogeography and Systematics of Mayflies and Stoneflies. (F.R. Hauer, J.A. Stanford \& R.L. Newell, eds.). University of California Press, Berkeley, p.125-132. http://dx.doi.org/10.1525/ california/9780520098688.003.0010

FROEHLICH, C.G. 2009. Plecoptera. In Macroinvertebrados bentónicos sudamericanos.( E. Domínguez \& H.R. Fernández, eds.). Fundación Miguel Lillo, Tucumán, cap.5, p.145-165.

MELO, A.S. \& FROEHLICH, C.G. 2001. Macroinvertebrates in neotropical streams: richness patterns along a catchment and assemblage structure between 2 seasons. J. N. Am. Benthol. Soc. 20(1):1-16. http://dx.doi.org/10.2307/1468184

MELO, A.S. \& FROEHLICH,C.G. 2004. Colonization by macroinvertebrates of experimentally disturbed stones in three tropical streams differing in size. Int. Rev. Hydrobiol. 89(3):317-325. http://dx.doi.org/10.1002/iroh.200310702 
Froehlich, C.G.

ROQUE, F.O., LECCI, S.L., SIQUEIRA, T. \& FROEHLICH, C.G. 2008. Using environmental and spatial filters to explain stonefly occurrences in southeastern Brazilian streams: implications for biomonitoring. Acta Limnol. Bras. 20(1):35-44.

Apêndice 3. Lista de trabalhos sobre Plecoptera do Estado de São Paulo anteriores a 2009.

Appendix 3. List of papers on Plecoptera from São Paulo State published before 2009.

FROEHLICH, C.G. 1969. Studies on Brazilian Plecoptera 1. Some Gripopterygidae from the Biological Station at Paranapiacaba, State of Sao Paulo. Beitr. Neotrop. Fauna 6:17-39.

FROEHLICH, C.G. 1984a. Brazilian Plecoptera 2. Species of the serrana-group of Kempnyia (Plecoptera). Aquat. Ins. 6(3):137-147. http://dx.doi. org/10.1080/01650428409361176

FROEHLICH, C.G. 1984b. Brazilian Plecoptera 3. Macrogynoplax veneranda sp.n. (Perlidae: Acroneuriinae). Ann. Limnol. 20(1-2):39-42. http://dx.doi. org/10.1051/limn/1984016

FROEHLICH, C.G. 1984c. Brazilian Plecoptera 4. Nymphs of perlid genera from southeastern Brazil. Ann. Limnol. 20(1-2):43-48. http://dx.doi.org/10.1051/ $\operatorname{limn} / 1984018$

FROEHLICH, C.G. 1988. Brazilian Plecoptera 5. Old and new species of Kempnyia (Perlidae). Aquat. Ins. 10(3):153-170. http://dx.doi. org/10.1080/01650428809361324

FROEHLICH, C.G. 1990. Brazilian Plecoptera 6. Gripopteryx from Campos do Jordão, State of São Paulo (Gripopterygidae). Stud. Neotrop. Fauna Environ. 25(4):235-247. http://dx.doi.org/10.1080/01650529009360823

FROEHLICH, C.G. 1993. Brazilian Plecoptera 7. Old and new species of Gripopteryx (Gripopterygidae). Aquat. Ins. 15(1):21-38. http://dx.doi. org/10.1080/01650429309361496

FROEHLICH, C.G. 1994. Brazilian Plecoptera 8. On Paragripopteryx (Gripopterygidae). Aquat. Ins. 16(4):227-239. http://dx.doi.org/10.1080/01650429409361559

FROEHLICH, C.G. 1996. Two new species of Kempnyia from southern Brazil (Plecoptera: Perlidae). Mitt. Schweizer. Ent. Ges. 69:117-120.

FROEHLICH, C.G. 1998. Seven new species of Tupiperla (Plecoptera: Gripopterygidae) from Brazil, with a revision of the genus. Stud. Neotrop. Fauna Environ. 33:19-36. http://dx.doi.org/10.1076/snfe.33.1.19.2170

ILLIES, J. 1963. Revision der südamerikanischen Gripopterygidae (Plecoptera). Mitt. Schweizer. Ent. Ges. 36:145-248.

NAVÁS, L. 1925. Familia Pérlidos. Insectos Neotrópicos (1ª serie). Rev. Chil. Hist. Nat. 29:305-313.

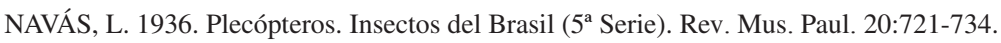

OLIVEIRA, L.G. \& FROEHLICH, C.G. 1998. Diversity and community structure of aquatic insects. (Ephemeroptera, Plecoptera and Trichoptera) in a mountain stream in southeastern Brazil. Acta Limnol. Bras. 9:139-148. 